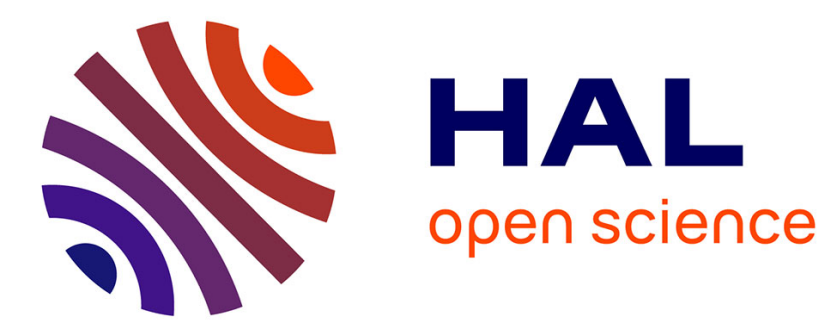

\title{
Evaluation of simulators for x-ray speckle-based phase contrast imaging
}

Laurene Quenot, Emmanuel Brun, Jean Michel Létang, Max Langer

\section{To cite this version:}

Laurene Quenot, Emmanuel Brun, Jean Michel Létang, Max Langer. Evaluation of simulators for x-ray speckle-based phase contrast imaging. Physics in Medicine and Biology, 2021, 66 (17), pp.1-12. 10.1088/1361-6560/ac1f38. hal-03329939

\section{HAL Id: hal-03329939 \\ https://hal.science/hal-03329939}

Submitted on 28 Oct 2021

HAL is a multi-disciplinary open access archive for the deposit and dissemination of scientific research documents, whether they are published or not. The documents may come from teaching and research institutions in France or abroad, or from public or private research centers.
L'archive ouverte pluridisciplinaire $\mathbf{H A L}$, est destinée au dépôt et à la diffusion de documents scientifiques de niveau recherche, publiés ou non, émanant des établissements d'enseignement et de recherche français ou étrangers, des laboratoires publics ou privés. 
Phys. Med. Biol.

\title{
Evaluation of simulators for X-ray speckle-based phase contrast imaging
}

\author{
L Quénot ${ }^{1}$, E Brun ${ }^{1}$, J M Létang ${ }^{2}$ and M Langer ${ }^{2,3}$ \\ ${ }^{1}$ Inserm UA7 Strobe, Université Grenoble Alpes, Grenoble France \\ ${ }^{2}$ Univ Lyon, INSA-Lyon, Université Claude Bernard Lyon 1, UJM-Saint Étienne, \\ CNRS, Inserm, CREATIS UMR 5220, U1206, F-69373, LYON, France \\ 3 TIMC-IMAG Laboratory (CNRS UMR 5525), Université Grenoble Alpes, \\ Grenoble, France \\ E-mail: jean.letang@creatis.insa-lyon.fr
}

May 2021

\begin{abstract}
X-ray phase contrast imaging (PCI) denotes a group of highly sensitive imaging techniques that permits imaging at scales ranging from nanoscopic to the medical. Recently introduced, speckle-based imaging has seen a rapid development because of its experimental simplicity and its capability to retrieve the refraction, the scattering and the absorption of a sample using a conventional X-ray set-up. Precise simulation would permit to optimise the imaging setups for different applications, but until now works on simulation of X-ray speckle-based phase contrast imaging have been very few. In this work we evaluate different simulation codes, based on Monte-Carlo, analytical ray-tracing and wave-optics Fresnel propagation. The simulation results are compared to both synchrotron and conventional imaging experiments to permits their validation. We obtain a strong similarity between simulated and experimental data. We discuss the validity and applicability of each approach.
\end{abstract}

Keywords: X-ray Phase Contrast Imaging, Speckle based Imaging, Monte-Carlo Simulations

\section{Introduction}

Since the seminal work of Roentgen, standard X-ray imaging is based on the same physical phenomenon: the attenuation by the tissues. But wave phenomena, the fact that x-rays are deviated when passing through matter, are under some conditions more dominant than attenuation. Indeed, the refraction index of material can be a thousand times greater (Bravin et al., 2013) than its counterpart attenuation factor for light elements. This translates into a much greater contrast for soft tissues with X-ray imaging methods based on the sensing of the phase (so-called Phase-Contrast Imaging (PCI) ) in comparison to the current traditional method based on attenuation. This property becomes highly interesting when one wants to image biological tissue with high resolution. Over the past few decades, an increasing number of studies 
have demonstrated the high diagnostic potential of PCI, as compared to conventional radiology, in a wide range of pathologies (Bravin et al., 2013). The simplest technique, discovered 1995, is achieved by simply letting the wave propagate in free space after interaction with a sample. Using a sufficiently coherent beam and X-ray optics, an ultra-microscope can be achieved that permits imaging at submicrometer resolutions using free space propagation, as shown by Mokso et al., 2007. On the other end of the spectrum, a technique discovered in 2008, using a random spatial beam modulator shows promise to bring X-ray phase contrast imaging to the clinic (Rougé-Labriet et al., 2020). The spatial modulator permits phase-contrast imaging with the use of a partially coherent source via the reconstruction of the sample refraction by tracking changes in the resulting "speckle" pattern (Cerbino et al., 2008; Berujon et al., 2012; Morgan et al., 2012).

Simulation has become increasingly important for the conception of imaging systems (Salvadori et al., 2020). Simulation of phase contrast imaging has proven to be challenging but would eventually permit for example: i) optimisation of imaging setups for different applications, from clinical imaging with conventional sources to high resolution imaging at synchrotron facilities, ii) optimisation of the techniques themselves, for example the scattering membranes used in speckle-based imaging or the imaging conditions used in propagation-based imaging, and iii) the development of new reconstruction algorithms, for example taking into account scattering. A simple analytical simulator based on wave optics can trivially be implemented using the Fresnel transform (Langer et al., 2008). While this kind of simulation reproduces well the effects of phase contrast, it seems difficult to correctly include other effects, such as reflection or scattering.

Contrary to the wave optics approach, Monte Carlo simulation is based on particle tracking and can correctly simulate attenuation, refraction, and scattering (Bottigli et al., 2004; Peter et al., 2014; Langer et al., 2020). Some methods to include phase contrast and coherent effects in Monte Carlo simulations have been proposed. Particle based simulations of X-ray phase contrast seems to have been first investigated by (Peterzol et al., 2005) who showed within which limits the ray-optical and wave-optical approaches are equivalent. The first Monte Carlo based simulation of X-ray phase contrast was presented by (Peter et al., 2014), who simulated propagation-based and grating-based phase contrast by simulating absorption, refraction, path length, and Compton scattering. Each particle is transformed to a wave through a heuristic, and the exit plane is binned to discretized images of the wave-field where contributions from each particle arriving in a bin is summed. The resulting wave is then propagated to the detector using the standard wave-optics approach. The Monte Carlo part was implemented using EGSnrc (Kawrakow et al., 2013). A seemingly identical approach, based on absorption and refraction with "time of flight" binning of the resulting wave-field and propagation using wave optics, was presented by Cipiccia et al., 2014. The Monte Carlo simulation was implemented using FLUKA (Bohlen et al., 2014). Scattering was not included in the model, however. The simulation of a double-slit 
experiment is presented, but does not seem to be capable of reproducing the photon by photon construction of the diffraction pattern. The same limitations apply as in Peter et al., 2014.

On the other end of the spectrum of simulation tools, a simple wave-optical simulator of X-ray phase contrast can trivially be implemented (Langer et al., 2008; Krivonosov et al., 2020), and the limit of ray tracing and wave-optical approaches have been investigated (Peterzol et al., 2007; Diemoz et al., 2014; Gureyev et al., 2017). Another limit of the classic wave-optical approach is that it assumes a thin object, meaning there are no secondary events. To overcome these limitations, multi-slice simulators have been proposed: the object is partitioned into several portions and the wave is propagated successively between the partitions, thus permitting some account for multiple interactions within the object, as well as for total reflection (Li et al., 2017; Shanblatt et al., 2019). This can be used for example in reconstruction algorithms beyond the depth of focus, important in X-ray nano-imaging (Ali et al., 2020). To the best of our knowledge only Zdora et al., 2015 simulated a speckle-based phase-contrast imaging (SBI) set-up using a simplistic Fresnel propagator approach.

In this work, we present three simulations methods and their associated results for the simulation of X-ray SBI: a Fresnel propagator, a home-made analytical ray tracing algorithm and a Monte-Carlo method implemented in Gate. Validation of those codes was performed with synchrotron and conventional source data in radiography mode. Finally a discussion on the limitation of each method is presented at the end of the article.

\section{Simulation methods}

\subsection{Ray-tracing}

The first simulation tool under consideration is based on a ray tracing model. We start by considering the projection approximation which is valid when the object is small in comparison to the propagation distance and states that the description of the wave through an object can be described as phase shift and that attenuation follows straight lines through the object. It means that we can calculate the phase shift $\Delta \phi$ and the attenuation $B$ through the entire object with the following relations:

$$
\begin{aligned}
& \Delta \phi(x, y)=-k \int_{z} \delta_{\lambda}(x, y, z) d z \\
& B(x, y)=k \int_{z} \beta_{\lambda}(x, y, z) d z
\end{aligned}
$$

where $x$ and $y$ are the spatial coordinates in the plane perpendicular to the propagation direction $z, \delta$ and $\beta$ are the indices for refraction and absorption of the materials calculated from the NIST database (Brennan et al., 1992), $\lambda$ is the wavelength and $k=2 \pi / \lambda$ is the wave number.

The intensity after the object can then be calculated as

$$
I_{\lambda, \text { after_object }}(x, y)=I_{\lambda, \text { before_object }}(x, y) \exp (-2 B(x, y)) \text {. }
$$


From the phase shift, the refraction angle of each ray can be computed as

$$
\alpha(x, y)=\frac{1}{k} \nabla(\Delta \phi(x, y))
$$

and using that angle map, the redistribution of intensities on the detector plane after propagation over a distance $z_{2}$ can be calculated as

$$
I_{\lambda, z_{2}}\left(x_{d}, y_{d}\right)=I_{\lambda, \text { after_object }}\left(x_{0}, y_{0}\right)
$$

where

$$
\left(x_{d}, y_{d}\right)=\left(x_{0}, y_{0}\right)+z_{2} \tan (\alpha(x, y)) .
$$

This model was also used in several x-ray phase contrast simulation studies such as that of Hassan et al., 2020.

For the polychromatic case, the spectrum of a tungsten anode is simulated using the python software toolkit SpekPy (Bujila et al., 2020). Then the intensity contributions of each of the energies were summed before simulating the detection. Since the incident spectrum of the laboratory experiment (see subsection 3.2) is in a low energy range, the assumption of total absorption in the scintillator seems justified to model the detector response (about $99 \%$ of the incident energy spectrum is absorbed by the CsI). Therefore, the detector quantum efficiency was considered to be constant and equal to one over the considered spectrum. For a higher tube voltage, such an assumption can no longer be made, and Monte Carlo simulations would need to be carried out to get a more realistic model of the detector response.

The detector pixel is over-sampled by a factor of $4 \times 4$ to get avoid aliasing artefacts. The intensity received in the detector plane is then convoluted with the source projected size and sampled at the detector pixel size. Then a convolution with the point spread function of the detector was applied. Finally, shot noise is modeled using a Poisson distribution for a more realistic result.

\subsection{Fresnel propagator}

The second simulation tool is based on the wave-optics model for x-rays, describing propagation as a linear operator usually called the Fresnel propagator. Wave descriptions for coherent x-rays have been developed and are detailed in the book of Paganin, 2006. The starting point of the model is to write the wave equation of the beam:

$$
\psi_{\lambda}=\exp \left(i\left(k_{x} x+k_{y} y+k_{z} z\right)\right)
$$

where $\lambda$ is the wavelength, $z$ is the coordinate along the propagation axis, $x$ and $y$ are the coordinates on the perpendicular plane, $k_{x}, k_{y}$ and $k_{z}$ are the components of the wave vector. Before the wave reaches any object, it can be considered that the wave vector is along the propagation direction therefore, equation 7 becomes:

$$
\psi_{\lambda}(x, y, z)=\exp \left(i k_{z} z\right)
$$


Then, when a wave goes through an object, it will be partly attenuated and phase shifted. A simple way to calculate those changes is to use the projection approximation as in equation 1. Under that approximation, the wave after the object will be modified by a transmittance function that can be described as follows:

$$
\psi_{\lambda}\left(x, y, z_{\text {after_object }}\right)=\mathcal{T}_{\text {object }} \psi_{\lambda}\left(x, y, z_{\text {before_object }}\right)
$$

where the transmittance function is

$$
\mathcal{T}_{\text {object }}=\exp (i \Delta \phi(x, y)-B(x, y)) .
$$

However this approximation has limits as it was shown by Morgan et al., 2010 and for larger objects or coherent sources, it may be biased.

Once the wave carries the information about the object, it will undergo further changes as it propagates to the detector plane. The experiments of interest here being mainly limited to the Fresnel domain under the paraxial approximation, this propagation can be described by the "Fresnel propagator" (Goodman, 2017; Paganin, 2006). There are two formulations of that propagator, one in the real space and the second one in the Fourier space. The first one including a convolution product, it has a high computation cost while the second one is a simple multiplication in the Fourier space as shown in equation 11

$$
\psi_{\lambda}\left(x, y, z_{\text {object }}+z_{2}\right)=D_{z_{2}}^{(F)} \psi_{\lambda}\left(x, y, z_{\text {object }}\right)
$$

where the Fresnel propagator is expressed in the diffraction operator $D_{z_{2}}^{(F)}$

$$
D_{z_{2}}^{(F)}=\exp \left(i k z_{2}\right) \mathcal{F}^{-1} \exp \left[-\frac{i z_{2}}{2 k}\left(k_{x}^{2}+k_{y}^{2}\right)\right] \mathcal{F}
$$

and $z_{2}$ is the distance of propagation.

After propagation to the detector plane, the wave function is converted to intensity information with the following relationship:

$$
I_{\lambda}(x, y, z)=\left|\psi_{\lambda}\left(x, y, z_{\text {detector }}\right)\right|^{2} .
$$

The polychromatic case was treated in the same way as for the ray tracing model. For all the previous steps, the working pixel is over-sampled by a factor of $4 \times 4$ compared to the detector pixel to avoid aliasing artefacts. Then, re-sampling and convolution with the source blurring and the detector's PSF were applied as well as the introduction of the shot noise the same way as for the ray-tracing code.

Those two approaches implemented in python3 are available at https://github. com/quenotl/PARESIS.

\subsection{Monte-Carlo}

The third simulation tool is based on Monte Carlo simulation and takes into account both the refraction and total reflection occurring in the object. The simulator is implemented with GATE v9.0 (Sarrut et al., 2014) which is based on Geant4 v10.6. The "emstandard_opt4" physics list builder has been set (Monarsh University model 
for Compton scattering and Livermore models for Photo-electric effect and Rayleigh scattering). This choice for the electromagnetic standard physics of Geant4 has been driven by a recent benchmarking report from Arce et al., 2020 on behalf of the Geant4 medical simulation benchmarking group which recommended the use "option 4". The differences between the physics models should in any case be marginal for our study because it is the refraction process which conditions the phase contrast in the MC simulation. We set the production threshold of gamma and electron to $1 \mu \mathrm{m}$. This might not have been optimized in terms of computing efficiency, but this threshold has been set in accordance with the scale of our setups: speckle grains a few micrometers in diameter, fibers a few tens of micrometers in diameter and pixel size as small as $3 \mu \mathrm{m}$ (for the synchrotron experiment).

Refractive indices are calculated using Xraylib (Schoonjans et al., 2011), integrated in GATE for this purpose (Langer et al., 2020). X-ray particles propagation is step-bystep in the usual Monte Carlo way. In addition, for every step of the particle propagation algorithm, the refractive index in the current step is compared to the refractive index in the previous step. If a change is detected, the particle is either refracted or reflected. The critical angle of incidence for reflection is calculated as

$$
\theta_{1}^{c}=\operatorname{asin}\left(\frac{1-\delta_{\lambda, 2}}{1-\delta_{\lambda, 1}}\right),
$$

where $\delta_{\lambda, 1}$ and $\delta_{\lambda, 2}$ are the refractive index decrements before and after the interface, respectively. If the angle of incidence is above the critical angle, the particle is reflected. If the angle of incidence is below the critical angle, the particle is refracted using Snell's law

$$
\left(1-\delta_{\lambda, 1}\right) \sin \theta_{1}=\left(1-\delta_{\lambda, 2}\right) \sin \theta_{2},
$$

where $\theta_{1}$ is the incident angle and $\theta_{2}$ is the refraction angle. The total number of particles per image is set to $2.5 \times 10^{9}$. The energy distribution of the x-ray source for the polychromatic case was set to the one used by the ray-tracing approach (computed using the python software SpekPy from Bujila et al., 2020). When a membrane is used for speckle-based imaging, the grains are modelled as sphere objects in GATE. The detector PSF and the Poisson noise are applied offline using standard Python routines from SciPy and NumPy, respectively. To make sure all three simulation images have same noise level we proceeded as follows:

- the initial intensity (leaving the source) in the ray-tracing approach was equivalent to the photon count used in Monte-Carlo simulation for a solid angle corresponding to the voxel size. Similarly the initial amplitude of the wave function of the Fresnel approach was calculated as the square root of this intensity as the final intensity detected is calculated with $I=\psi^{2}$.

- the Poisson noise level was then set according to this photon count and eventually checked for the three simulations methods by measuring the standard deviation on windows outside the sample on propagation images. 


\section{Experimental measurements}

\subsection{Membrane microscopic quantification}

Speckle-based imaging is usually performed with sandpaper sheets and in such configuration the grains are poly-disperse both in shape and size. Moreover such material is limited to synchrotron applications and cannot be used on conventional X-ray source to create a random intensity modulation. Indeed silicon, the heaviest component of sandpaper, is of too low atomic number to create a modulation in intensity based only on absorption with a conventional source with a very limited coherence. For all these reasons we used a metallic powder, CuSn (provided by GoodFellow UK). Despite a good calibration of the grain size we analysed the grain size on microscopic plate with the same process we use to create our membrane for the X-Ray. The image of the whole membrane was obtained with a translation motor plate on an Olympus BX 50 microscope. The lens used was a $\times 5$ resulting pixel size measured at $0.65 \mu \mathrm{m}$. The total field of view imaged by the microsocope was $7.4 \mathrm{~mm} \times 4.5 \mathrm{~mm}$. Thanks to the relatively small size of the grain, the images were sharp and the focal plane was thick enough to get focus on the whole grains.

The quantification of the microscopic images was made using the software iMorph (Brun et al., 2008). After binarization of the image (threshold obtained by Otsu algorithm) we computed a meshing by circles of the CuSn grains. A modified version of the algorithm published in (Brun et al., 2017) was elaborated on purpose for this study. Briefly, the algorithm starts by computing the distance map Dist of the binary image, then uses a heap to sort every pixel by their distance to border value. After this step, every pixel $p$ of the heap is processed in the same way: The discrete disk centered in $p$ with a radius equal to $\operatorname{Dist}(p)$ is computed. If a pixel belonging to this disk was already validated in the result image the whole disk is discarded, otherwise the whole disk is validated. This does not compute a perfect meshing of the surface but is a reasonable first approximation for quasi spherical grains. The figure 3.1 shows the results of this meshing. Once the mesh was obtained the center of disks was reported together with the their radius in a file used for the simulation of the membrane with all the different methods.

\subsection{Validation experiments}

In order to study the performance of the three simulators, two experiments of SBI were performed (cf experimental set-up on Figure 2). One monochromatic at the BioMedical Beamline (ID17) of the European Synchrotron Radiation Facility (ESRF) and the second polychromatic on a laboratory set-up EASYTOM XL (RX Solutions, France) at SIMAP (Grenoble):

(i) The ID17 experiment is using monochromatic synchrotron radiation at $52 \mathrm{keV}$. The CuSn membrane was placed $144 \mathrm{~m}$ away from the source. The sample, a $70 \mu \mathrm{m}$ radius nylon wire, was placed $1.6 \mathrm{~m}$ after the membrane and the detector $3.6 \mathrm{~m}$ 

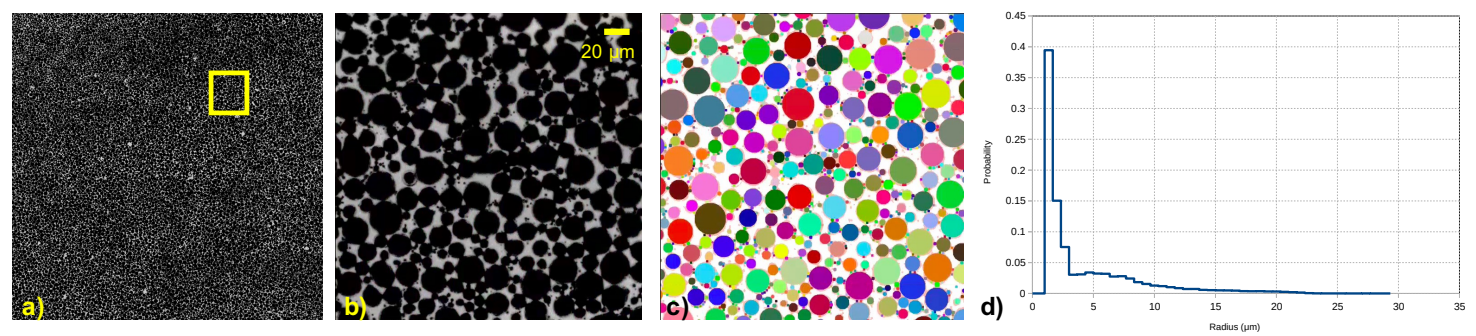

Figure 1. Modeling the random mask membrane used for simulation: a) microscopic image of the whole membrane obtained with $a \times 5$ lens $b$ ) inset in the image c) meshing by sphere of the inset zone obtained by the iMorph software d) granulometry obtained on the whole image.

away from the sample. The detector was a sCMOS with a pixel size of $3 \mu \mathrm{m}$ and a PSF modeled by a Gaussian of standard deviation 2.5 pixels. Five different membrane positions - simple translations in the $(x, y)$ plane - were used for the phase retrieval. The Unified Modulated Pattern Analysis (UMPA) proposed by Zdora et al., 2017 was chosen to retrieve the phase because it does not require a large number of membrane positions nor any assumption on the sample.

(ii) The laboratory experiment was performed using a tungsten anode at $40 \mathrm{kVp}$ with a $200 \mu \mathrm{m}$ thick beryllium exiting window. The distance from source to membrane was $41.5 \mathrm{~mm}$, membrane to sample $21.5 \mathrm{~mm}$ and sample to detector $607 \mathrm{~mm}$. The sample was also a Nylon wire but with a $100 \mu \mathrm{m}$ radius. The detector was a Varian flat panel with a pixel size of $127 \mu \mathrm{m}$ and a PSF modeled by a Gaussian of standard deviation 1.2 pixels and protected by a $2.5 \mathrm{~mm}$-thick carbon-fiber plate. All elements as well as the air volume were considered for the polychromatic simulations.
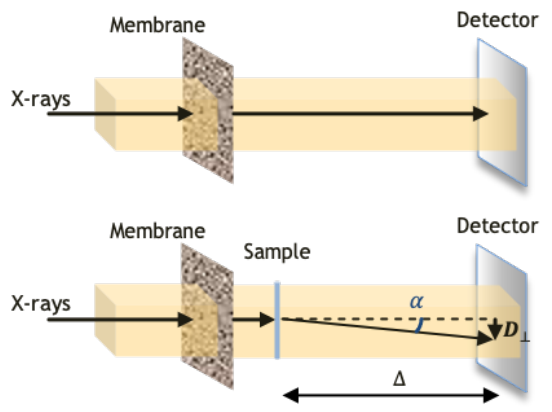
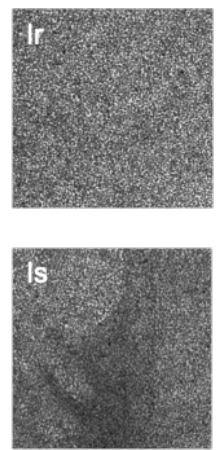



Figure 2. Left, speckle-based imaging set-up: reference image (Ir) acquisition with membrane but without the sample and sample image (Is) acquisition with the sample. Right, tungsten anode spectrum at $40 \mathrm{kVp}$ obtained with Spekpy.

In both experiments, simple propagation images (i.e. same geometries but without the speckle membrane) have also been acquired. 


\subsection{Similarity measure}

The normalized root-mean-square error (NRMSE) is a standard measure and produces a nondimensional statistic which is very convenient to compare different datasets. Several normalizations exist, the most common ones are the range of the measured data and the mean value of the measurements. In our case where the data to be compared are in the projection domain, what is relevant for normalisation here is the contrast of the phase fringe and not the mean measure, for it is about one (because projections are flat-field corrected). We therefore calculated the normalized root mean square error as follows:

$$
\mathrm{NRMSE}=\frac{\mathrm{RMSE}}{\max \left(I_{\exp }\right)-\min \left(I_{\exp }\right)}
$$

between the experimental profile and the simulated profile.

\section{Results}

\subsection{Simple propagation using monochromatic synchrotron radiation}

A first simple propagation image of a Nylon wire was acquired to assess whether the three simulators gave results consistent with the experiment. The experimental setup was the one described in Section 3.2 for the ID17 experiment, but without the membrane in the path of the beam. Phase-contrast images are shown in Figure 3. Qualitatively, the simulated images (Figure $3(\mathrm{~b}-\mathrm{d})$ ) are quite similar to the experimental image $(3(\mathrm{a}))$. Profiles perpendicular to the wire were extracted from the images and are displayed in Figure 4. The similarity between the simulated and experimental profiles confirms that the three methods give results in agreement with the experiment. In the wire region the NRMSE is about $13 \%$ for each of the three simulators.
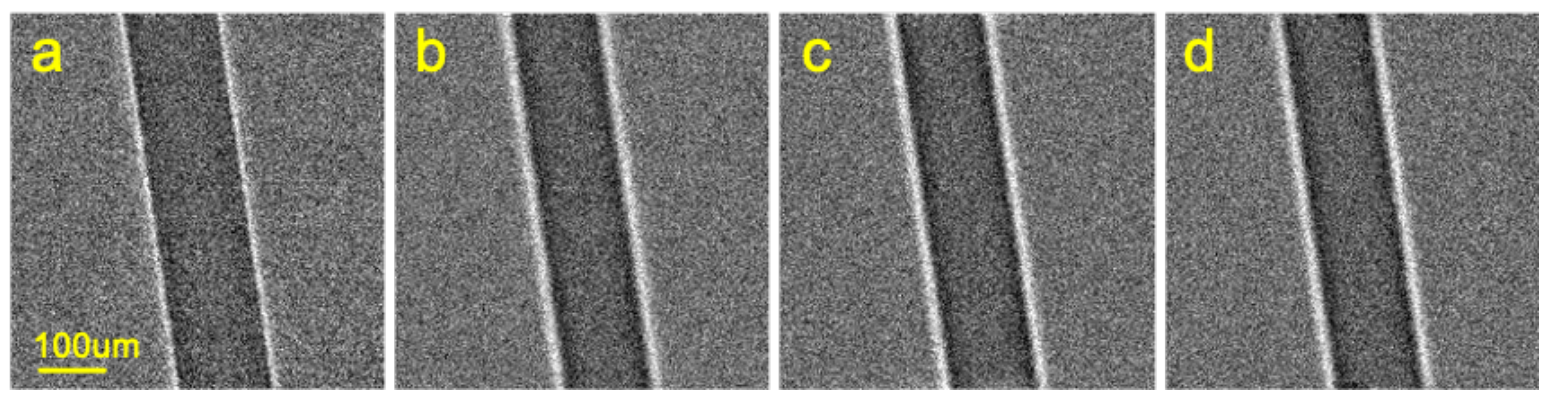

Figure 3. Propagation images of a Nylon wire from (a) ID17 experiment, (b) Monte Carlo (MC) simulation, (c) Fresnel propagator simulation, (d) Ray-tracing (RT) simulation.

\subsection{Speckle-based imaging using monochromatic synchrotron radiation}

The experimental setup remains the same as in the previous subsection, the speckle membrane is now inserted in the beam. Images of the speckle-based experiment were 


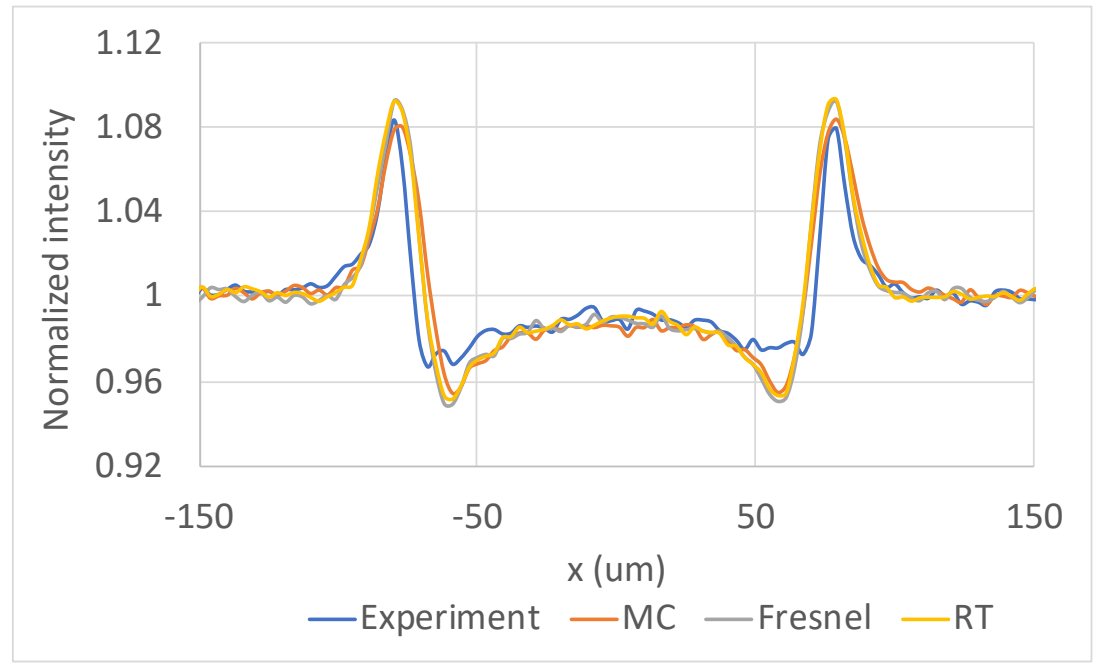

Figure 4. Profiles from the images of Figure 3 averaged over one hundred lines perpendicular to the wire.

then simulated with the three methods defined in section 2 from the parameters of the segmented membrane.
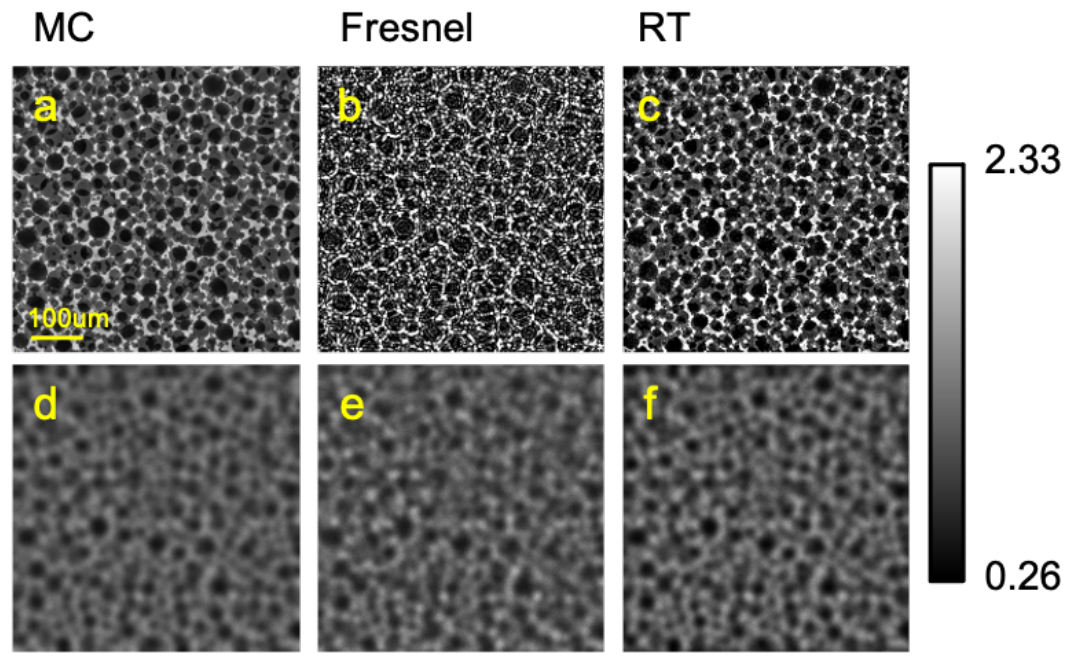

Figure 5. Simulated reference images of the membrane with Monte Carlo (MC), Fresnel propagator and ray-tracing (RT) methods without taking into account the PSF of the system (a-c), and with taking it into account (d-e).

Figure 5 presents the images obtained for the reference image (only the membrane in the path of the beam) with each simulation method, taking into account the detector PSF or not. We can see that without the PSF, the Fresnel propagator simulation gives results that are more contrasted than the others. This is due to the fact that this model calculates the interference fringes due to the coherence of the system which are not taken into account in the other models. Considering the actual experimental 
parameters, however, the blurring caused by the PSF makes the results from the different simulators very similar. The additional information accessible with the Fresnel model has very low visibility.

Figure 6 presents simulated sample images (Is) and reference images (Ir) and the ratio Is/Ir, along with the corresponding experimental images. The nylon wire is not directly visible in the sample images because its absorption is very weak compared to the membrane grains (see also the extracted profiles in Figure 7). We observe that the simulated images are very similar for the three models (NRMSE $\approx 15 \%$ ). The figure also shows that while the size of the grains in the simulated images is comparable to the experimental images, they appear less contrasted in the experimental images. This could be due to experimental parameters that are difficult to include in the simulations, such as the micro-structure of the membrane that acts as a substrate for the grains.

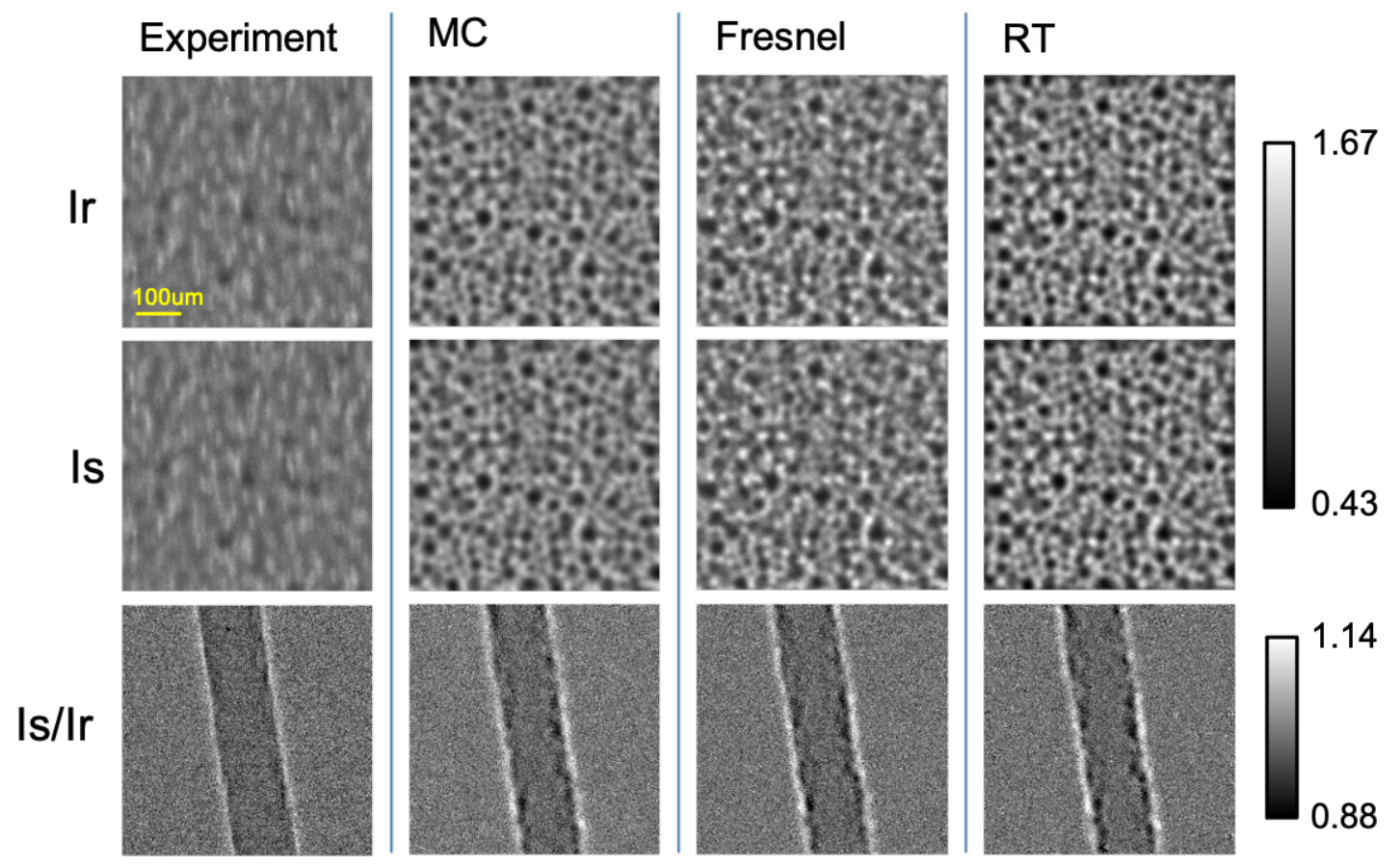

Figure 6. Sample and reference images from the synchrotron (ID17, ESRF) experiment with monochromatic illumination, and simulations obtained with the three different simulation codes, as well as the corresponding sample over reference ratio images.

\subsection{Phase retrieval from monochromatic synchrotron radiation}

To study the capability of the simulations to reproduce phase retrieval results, Figure 8 shows the estimated phase information from the experiment and all three simulators. The colour-coded direction together with the horizontal and vertical components are depicted. Figure 8 shows that the three simulation methods allow to retrieve the displacement vectors with rather similar results. We can see that the experimental 


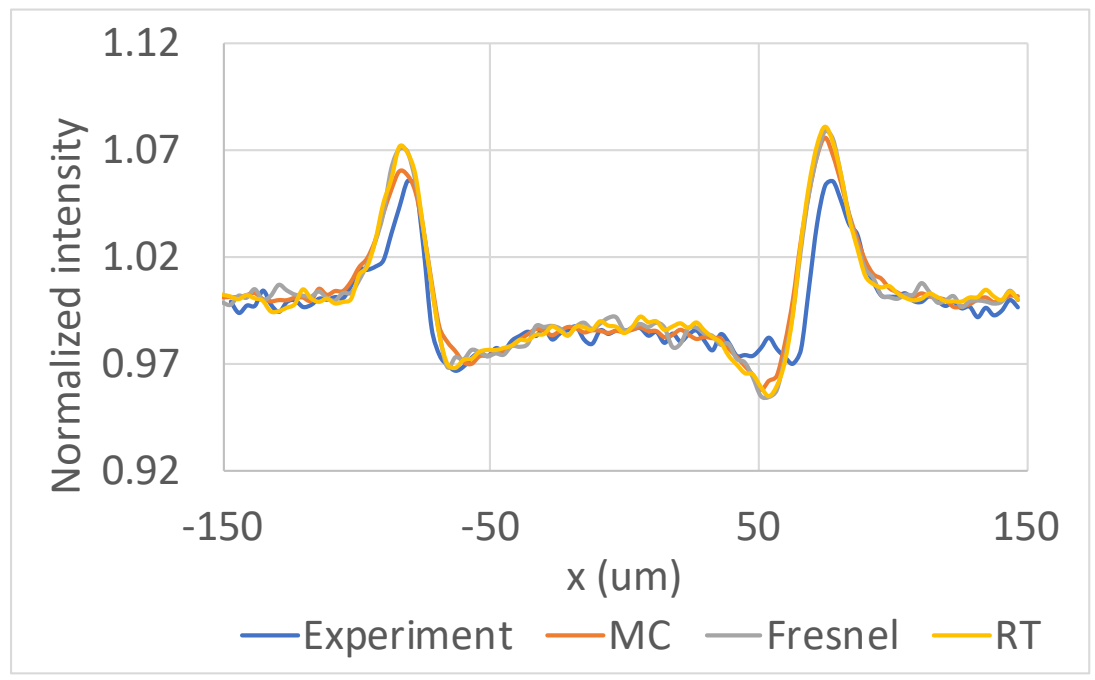

Figure 7. Profiles from the Is/Ir images of Figure 6 averaged over one hundred lines perpendicular to the wire.

result is a little bit noisier than the simulations. This phenomenon can be explained by the weaker contrast that was already noticed in the Is and Ir images. However, the correspondence is visually very good. The profiles extracted from the Dy images and displayed on Figure 9 confirms a very good agreement between the simulations and the experiment. The experimental profile seem to present a slight positive offset (0.05 pix). This can be due to a small displacement of the whole membrane between the sample and reference acquisitions of one or several pairs.

\subsection{Speckle-based imaging using a polychromatic laboratory source}

In addition to the monochromatic synchrotron experiment, the simulations were benchmarked against a polychromatic x-ray tube experiment. For this simulation setup, several experimental parameters had to be taken into account, especially the beam hardening due to the absorption of the lower energies in the air, in the source filter and in the carbon protective plate of the detector. Results with and without the detector PSF are presented in Figure 10. Qualitatively, the simulated images are very close to the experiment, especially when taking into account the detector PSF. Figure 11 presents the profiles of the wire extracted from the propagation images. We observe once again that the results obtained for the three simulations are very similar and very close to the experiment once the PSF is taken into account: the NRMSE in the wire region is about $8 \%$ for each of the three simulators with respect to the experimental profile.

\section{Discussion and conclusion}

With the objective of studying and optimising speckle-based imaging experiments, three simulation tools were developed and compared. The Monte-Carlo approach 


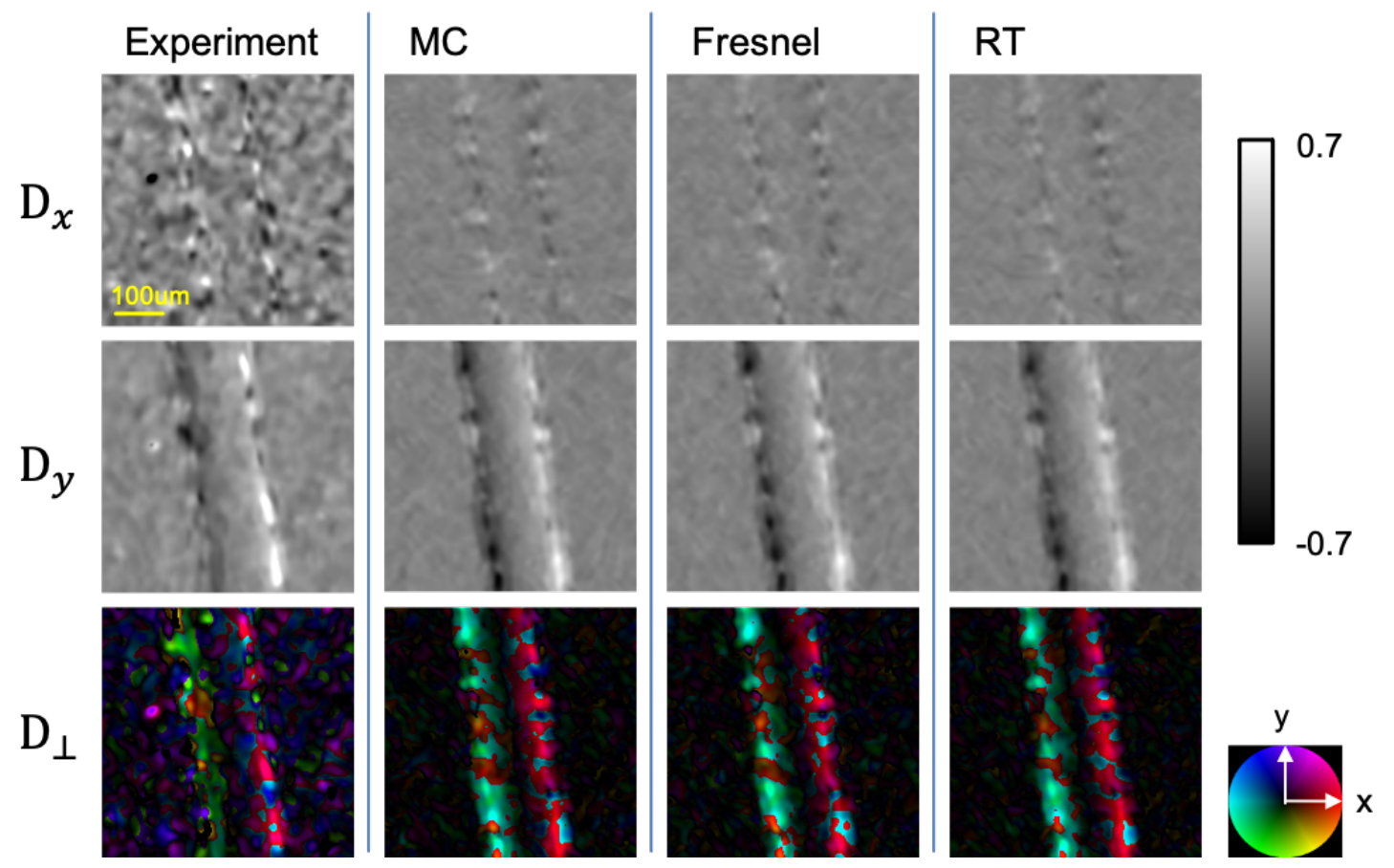

Figure 8. Displacements along the $\mathrm{x}$ axis $\left(D_{x}\right)$ and $\mathrm{y}$ axis $\left(D_{y}\right)$ retrieved from 5 pairs of sample and reference images. Coloured representation of the displacement vectors $\left(D_{\perp}\right)$.

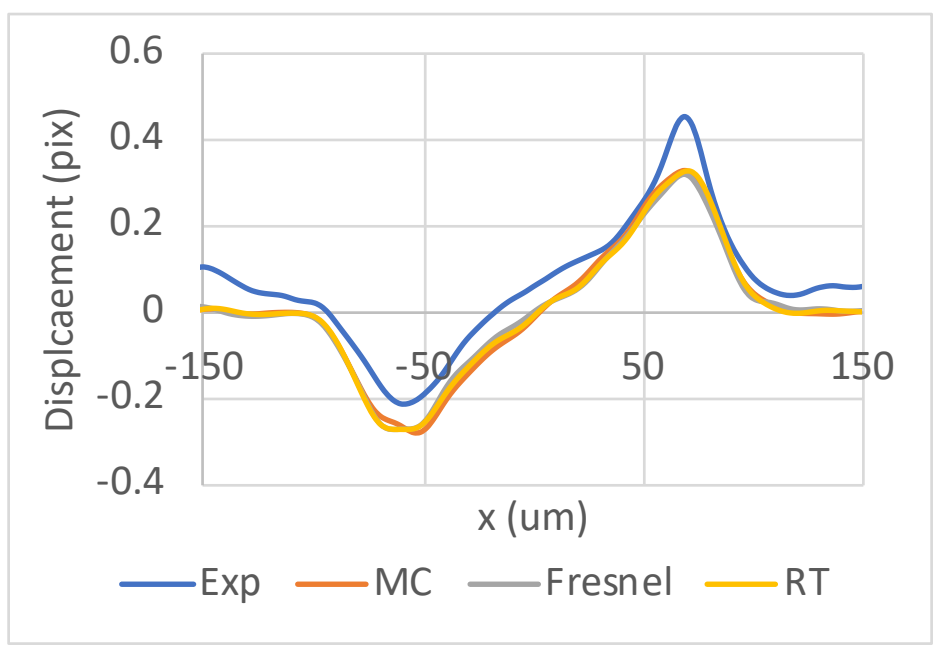

Figure 9. Profiles from the Dy images of Figure 8 averaged over one hundred lines perpendicular to the wire.

was implemented with the GATE software, taking into account absorption, scattering, refraction and total reflection. The two other models were implemented in Python. One is based on a ray-tracing model that takes into account only the absorption and the refraction of the beam. The other is based on a Fresnel propagator which allows to take into account the interference fringes due to partial coherence of the systems. 


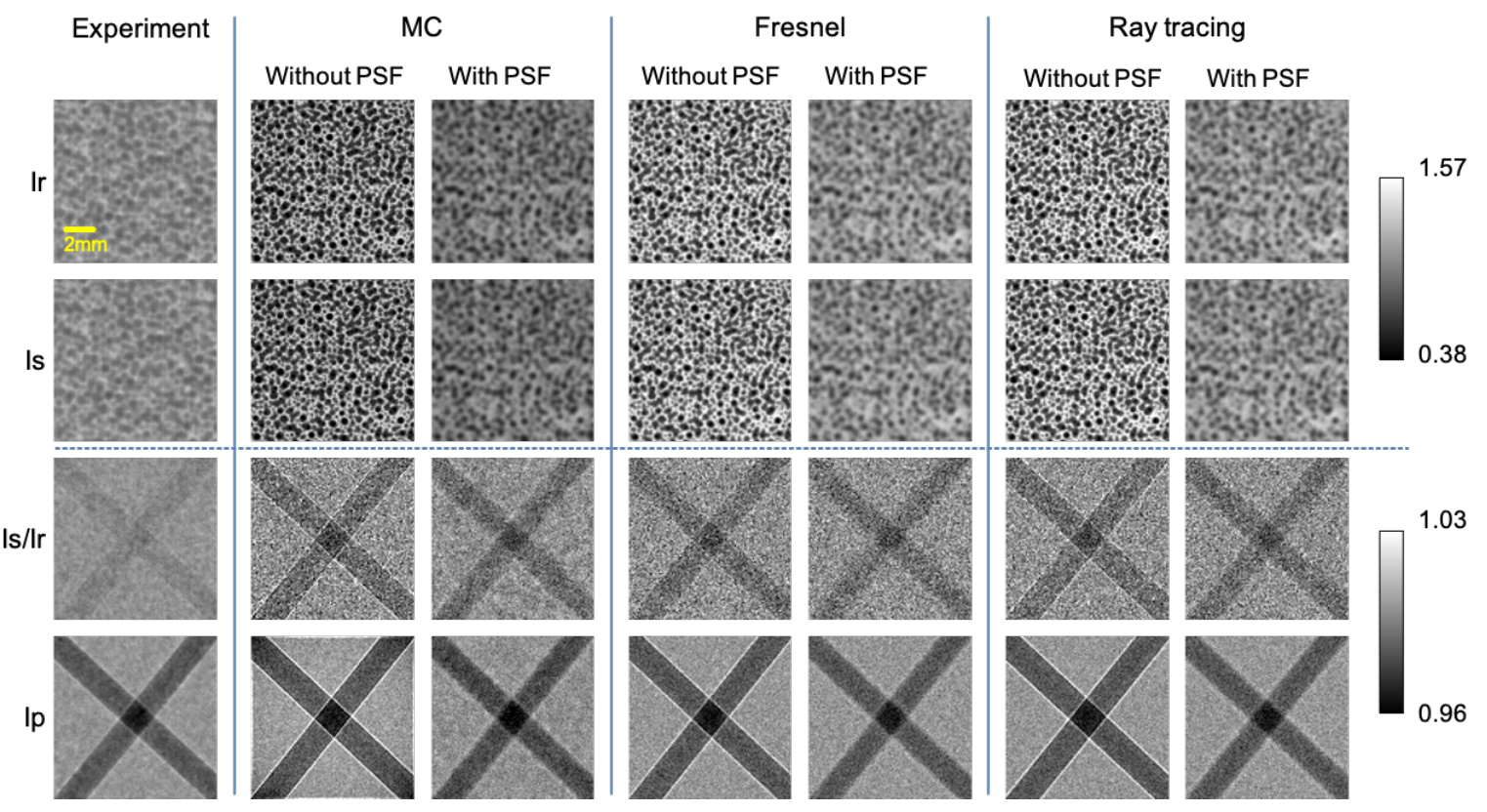

Figure 10. Sample and reference images (Is, Ir) from the laboratory experiment using a polychromatic source, and simulations obtained with the three different simulation codes, with and without taking into account the detector PSF. Sample over reference ratio images (Is/Ir) and simple propagation images (Ip).

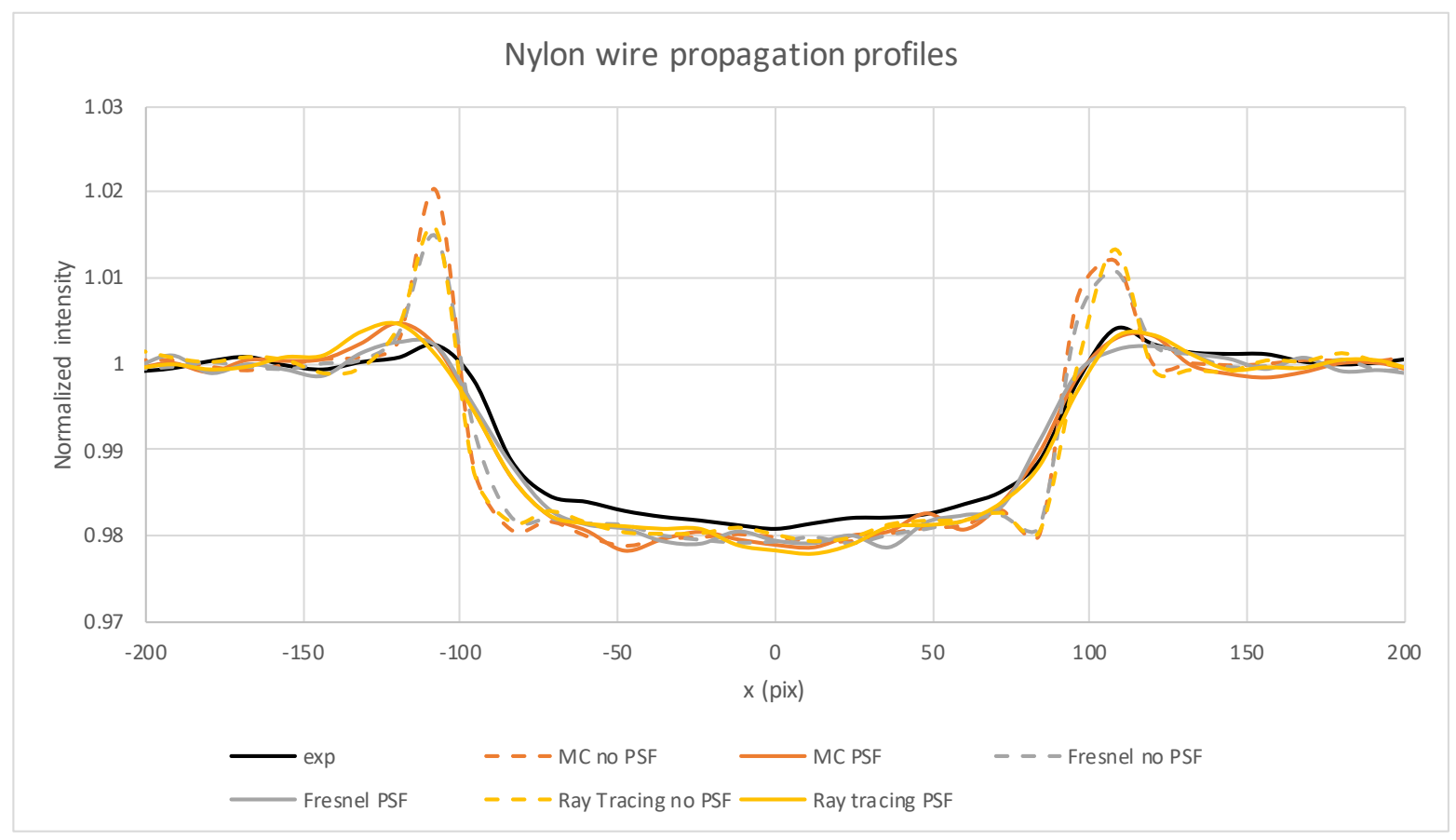

Figure 11. Profiles extracted from the propagation images (Ip) of Figure 10 averaged on 30 lines perpendicular to one of the wires. 
The phase fringes are visible in the Fresnel-simulated reference image without PSF (Figure 5.b) for the ID-17 experiment but not for the laboratory experiment (Figure 10). Both experiments are in the "edge-enhancement" regime and this is confirmed by

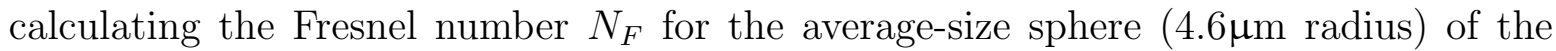
speckle membrane. We have $N_{F}=0.70$ for the monochromatic ID17 experiment and $N_{F} \in[7,70]$ for the polychromatic laboratory experiment. We also have a lower visibility of the edge enhancement of the wires in the laboratory experiment: the phase fringe for the wires in the laboratory experiment (Figure 10) is indeed much less visible than in the ID-17 experiment (Figure 6). This could be expected from the higher Fresnel number, the effect of the polychromatic illumination on the interference and the fact that the width of Fresnel fringes is related to $\sqrt{\lambda \Delta z}$. This gives $\sqrt{\lambda \Delta z}=3$ pixels for the ID-17 experiment and $\sqrt{\lambda \Delta z} \in[0.1,0.3]$ pixels for the laboratory experiment. A larger propagation distance or a smaller pixel size would have been required to get more a pronounced phase-contrast image for the laboratory experiment. The laboratory experiment was actually limited by the available characteristics of the used conventional CBCT system, the source size was set to its minimal size and the distance to the maximal possible offered by the system.

In the speckle-based imaging experiments studied here, all three models yield very similar results for the wires, and all very close to the experimental images. This might be somewhat surprising since the physics models differ in the three simulation methods. The source of phase contrast in both Monte Carlo and ray-tracing based simulations is the same, i.e. refraction, and the additional secondary photons in Monte Carlo (fluorescence and scatter) do not affect much the image since the propagation distance is very large. For the Fresnel-based simulation, we recall that the limit of the ray optical validity (Peterzol et al., 2005) states that

$$
\pi \lambda \Delta_{z} M\left(\frac{1}{2 \mathrm{FWHM}}\right)^{2} \ll 1
$$

where $M$ is the magnification and FWHM the full width half maximum of the detector PSF. This condition is met in both cases - namely 0.27 and between $[0.001,0.01]$ for the ID-17 and laboratory experiments, respectively - which means that the phase contrast in the Fresnel-based simulation with PSF will appear as the Laplacian of the phase shifts. This is why the Fresnel-simulated images give similar results as the other two simulation methods here.

It is interesting to note that all three models have their strengths and weaknesses. On one hand, the Monte-Carlo model is very computationally expensive since it calculates individually the path of each photon (about $150 \mathrm{~h}$ total computation time was needed for simulating a speckle-based image with $2 \times 10^{9}$ particles on a computing center). On the other hand, it can take into account a broad range of interactions including scattering and reflection and it will be the most accurate when simulating images with low statistics. Polychromatic imaging can also be modelled without any additional computation. These properties makes the Monte-Carlo technique particularly interesting for example for the simulation of spectral imaging combined with SBI. 
Conversely, the ray-tracing model allows a faster calculation since it only requires one path calculation for each sub-pixel in an over-sampled image. It cannot generate contrast from reflection or interference fringes, but for many systems the contrast it can provide is still consistent. This is the case for example for imaging in the "edgeenhancement" regime (where the Fresnel number is close to 1) where the resolution and propagation distance is relatively low, and for polychromatic sources and lower spatial coherence, where contrast from interference fringes will be weak.

Finally, the Fresnel propagator model is the fastest since the propagation calculation is treated as a multiplication in the Fourier space, thus only requiring two FFTs per wavelength for its computation. Note however that, contrary to the Monte Carlo simulator, both ray-tracing and Fresnel simulators require an external loop over the energies of the source, which means that their efficiency is dependent on the desired sampling of the spectrum. The Fresnel propagator model allows to take into account interference fringes along with the attenuation. As shown in the results, the additional information given by the interference fringes is mostly lost (both setups are within the validity limits of the ray-optical approach) due to the low resolution and coherence in the experiments performed here, but it might be an interesting tool for very high resolution imaging with SBI. It is also worth noting that the simulation of grating-based interferometry for phase contrast is compatible with the Fresnel propagator method, since it is based on the computation of the complex wavefront. The MC and raytracing methods however would not be able to simulate the Talbot intensity patterns unless a dedicated wavefront construction is implemented, as for example in the study of Sanctorum et al., 2020.

The most likely cause of the obvious discrepancies between the measured profiles and the simulated ones comes from the lack of a realistic surface modeling. All three simulations considered perfectly smooth cylinders for the Nylon fibers. The microscopic roughness of the surface has not been taken into account: the contrast of the simulated phase fringe is therefore likely to be overestimated. We made the assumption that the effect of the surface roughness could be modeled as a Gaussian blurring of the projection image. Therefore the detector PSF was tuned to recover the correct contrast of the phase fringes. A more appropriate formalism based on the statistical description of the interfaces is definitively required to improve the realism of the phase contrast fringes (Als-Nielsen et al., 2011).

Although scattering contributions are included in Monte Carlo simulation codes, their implementations are usually based on the independent atom approximation. This was the case in our Gate simulations. Paternò et al., 2018 recently implemented in Geant4 (releases 10.7 and later) inter-atomic interference effect in small-angle coherent Rayleigh scattering. This extension will be important to simulate more realistic images, in particular in view of dark-field contrast simulations.

We have presented an evaluation and validation of three different simulation tools for the simulation of SBI phase contrast. The validation of the simulation tools presented here will enable their use for the optimisation of the experimental parameters in SBI. 
Considering the increasing interest of dark-field imaging, it would also be interesting to incorporate the ultra-small x-ray scattering (if possible) into the different models. Finally, it would also be very interesting to further evaluate the simulators in a careful experimental study on biological samples.

\section{Acknowledgements}

This work was performed within the framework of the LABEX PRIMES (ANR-11LABX-0063) of Université de Lyon, within the program 'Investissements d'Avenir' (ANR-11-IDEX-0007) operated by the French National Research Agency (ANR). ESRF for beamtime, Simap for granting beatime on tomograph, R. Granger, L. Salvo P. Lhuissier for their help during the conventional source experiment. We also gratefully acknowledge the support from the CNRS/IN2P3 Computing Center (Lyon/Villeurbanne

- France), for providing a significant amount of the computing resources needed for this work.

\section{References}

Ali, S et al. (2020). "Comparison of distributed memory algorithms for X-ray wave propagation in inhomogeneous media". In: Opt. Express 28.20, pp. 29590-29618. DOI: $10.1364 / 0 E .400240$.

Als-Nielsen, J et al. (2011). Elements of Modern X-Ray Physics. John Wiley \& Sons. 436 pp. ISBN: 0470973943.

Arce, P et al. (2020). "Report on G4-Med, a Geant4 benchmarking system for medical physics applications developed by the Geant4 Medical Simulation Benchmarking Group". In: Medical Physics. DOI: 10.1002/mp.14226.

Berujon, S et al. (2012). "X-ray multimodal imaging using a random-phase object". In: Physical Review A 86.6, p. 063813. DOI: 10.1103/PhysRevA.86.063813.

Bohlen, T T et al. (2014). "The FLUKA code: developments and challenges for high energy and medical applications". In: Nuclear Data Sheets 120, pp. $211-214$. DoI: 10.1016/j.nds. 2014.07.049.

Bottigli, U et al. (2004). "Voxel-based Monte Carlo simulation of X-ray imaging and spectroscopy experiments". In: Spectrochimica Acta Part B: Atomic Spectroscopy 59.10, pp. 1747 -1754. DOI: 10.1016/j.sab.2004.03.016.

Bravin, A et al. (2013). "X-ray phase-contrast imaging: from pre-clinical applications towards clinics". In: Physics in Medicine and Biology 58.1, R1-35. DOI: 10.1088/ 0031-9155/58/1/R1.

Brennan, S et al. (1992). "A suite of programs for calculating x-ray absorption, reflection, and diffraction performance for a variety of materials at arbitrary wavelengths". In: Review of scientific instruments 63.1, pp. 850-853. DOI: 10.1063/1.1142625. 
Brun, E et al. (2008). "IMorph: A 3D morphological tool to fully analyse all kind of cellular materials". In: CellMet'08, Cellular Metals for Structural and Functional Applications, Dresden.

Brun, Emmanuel et al. (2017). "Fast Granulometry Operator for the 3D Identification of Cell Structures". In: Fundamenta Informaticae 155.4, pp. 363-372. DOI: 10.3233/ FI-2017-1590.

Bujila, R et al. (2020). "A validation of SpekPy: A software toolkit for modelling X-ray tube spectra". In: Physica Medica 75, pp. 44-54. DOI: 10.1016/j .ejmp. 2020.04 . 026.

Cerbino, R et al. (2008). "X-ray-scattering information obtained from near-field speckle". In: Nature Physics 4, pp. 238-243. DOI: 10.1038/nphys837.

Cipiccia, S et al. (2014). "Inclusion of coherence in Monte Carlo models for simulation of x-ray phase contrast imaging". In: Opt. Express 22.19, pp. 23480-23488. DOI: 10.1364/OE. 22.023480.

Diemoz, P C et al. (2014). "On the origin of contrast in edge illumination X-ray phasecontrast imaging". In: Optics Express 22.23, p. 28199. DOI: 10.1364/oe.22.028199.

Goodman, J W (2017). Introduction to Fourier Optics. W H Freeman \& Co. 564 pp. ISBN: 1319119166.

Gureyev, T E et al. (2017). "On the "unreasonable" effectiveness of transport of intensity imaging and optical deconvolution". In: Journal of the Optical Society of America A 34.12, p. 2251. DOI: 10.1364/josaa.34.002251.

Hassan, L et al. (2020). "Development of simulations for a mesh-based x-ray phase imaging system". In: Computational Imaging V. Ed. by Lei Tian et al. Vol. 11396. International Society for Optics and Photonics. SPIE, pp. 56 -65. DOI: 10.1117/ 12.2558926.

Kawrakow, I et al. (2013). The EGSnrc code system: Monte Carlo simulation of electron and photon transport. Tech. rep. NRCC PIRS-701. National Research Council, Ottawa, Canada. DOI: 10.4224/40001303.

Krivonosov, Y S et al. (2020). "Phase-Contrast Imaging in a Polychromatic X-ray Beam at a Laboratory Source". In: Crystallography Reports 65.4, pp. 503-507. DOI: 10 . $1134 / \mathrm{s} 1063774520040136$.

Langer, M et al. (2008). "Quantitative comparison of direct phase retrieval algorithms in in-line phase tomography". In: Medical Physics 35.10, pp. 4556-4566. Dor: 10 . $1118 / 1.2975224$.

Langer, M et al. (2020). "Towards Monte Carlo simulation of X-ray phase contrast using GATE". In: Optics Express 28.10, p. 14522. DOI: 10.1364/oe. 391471.

$\mathrm{Li}, \mathrm{K}$ et al. (2017). "Multislice does it all calculating the performance of nanofocusing X-ray optics". In: Opt. Express 25.3, pp. 1831-1846. DOI: 10.1364/OE. 25.001831. Mokso, R et al. (2007). "Nanoscale zoom tomography with hard x rays using KirkpatrickBaez optics". In: Applied Physics Letters 90.14, p. 144104. DOI: 10 . 1063/1. 2719653. 
Morgan, K S et al. (2010). "The projection approximation and edge contrast for x-ray propagation-based phase contrast imaging of a cylindrical edge". In: Opt. Express 18.10, pp. 9865-9878. DOI: 10.1364/OE.18.009865.

Morgan, K S et al. (2012). "X-ray phase imaging with a paper analyzer". In: Applied Physics Letters 100.12, p. 124102. DOI: 10.1063/1.3694918.

Paganin, D M (2006). Coherent X-Ray Optics. Oxford University Press, Oxford. IsBN: 9781429421607.

Paternò, G et al. (2018). "Geant4 implementation of inter-atomic interference effect in small-angle coherent X-ray scattering for materials of medical interest". In: Physica Medica 51, pp. 64-70. DOI: 10.1016/j.ejmp.2018.04.395.

Peter, S et al. (2014). "Combining Monte Carlo methods with coherent wave optics for the simulation of phase-sensitive X-ray imaging". In: Journal of Synchrotron Radiation 21.3, pp. 613-622. DOI: 10.1107/S1600577514000952.

Peterzol, A et al. (2005). "The effects of the imaging system on the validity limits of the ray-optical approach to phase contrast imaging". In: Medical Physics 32.12, pp. 3617-3627. DOI: 10.1118/1.2126207.

Peterzol, A et al. (2007). "X-ray phase contrast image simulation". In: Nuclear Instruments and Methods in Physics Research B 254.2, pp. 307 -318. DOI: 10 . 1016/j.nimb. 2006.11.042.

Rougé-Labriet, H et al. (2020). "X-ray Phase Contrast osteo-articular imaging: a pilot study on cadaveric human hands". In: Scientific Reports 10.1. DOI: 10 . 1038 / s41598-020-58168-3.

Salvadori, J et al. (2020). "Monte Carlo simulation of digital photon counting PET". In: EJNMMI Phys. 7, p. 23. DOI: 10.1186/s40658-020-00288-w.

Sanctorum, J et al. (2020). "X-ray phase contrast simulation for grating-based interferometry using GATE". In: Optics Express 28.22, p. 33390. DOI: 10.1364/ oe. 392337.

Sarrut, D et al. (2014). "A review of the use and potential of the GATE Monte Carlo simulation code for radiation therapy and dosimetry applications". In: Medical Physics 41.6, p. 064301. DOI: 10.1118/1.4871617.

Schoonjans, T et al. (2011). "The xraylib library for X-ray - matter interactions. Recent developments". In: Spectrochimica Acta Part B: Atomic Spectroscopy 66.11-12, pp. 776-784. DOI: 10.1016/j.sab.2011.09.011.

Shanblatt, E R et al. (2019). "Forward model for propagation-based x-ray phase contrast imaging in parallel- and cone-beam geometry". In: Opt. Express 27.4, pp. 45044521. DOI: $10.1364 / 0 \mathrm{E} .27 .004504$.

Zdora, M-C et al. (2015). "Simulations of x-ray speckle-based dark-field and phasecontrast imaging with a polychromatic beam". In: Journal of Applied Physics 118.11, p. 113105. DOI: 10.1063/1.4931145.

Zdora, M-C et al. (2017). "X-ray phase-contrast imaging and metrology through unified modulated pattern analysis". In: Physical Review Letters 118, p. 203903. DOI: 10 . 1103/physrevlett. 118. 203903. 